\title{
Eficiência bioeconômica de estratégias de alimentação em sistemas de produção de leite. 1. Produção por animal e por área1
}

\section{Francisco Palma Rennó ${ }^{*}$, José Carlos Pereira ${ }^{3}$, Carlos Antônio Moreira Leite ${ }^{4}$, Marcelo Teixeira Rodrigues ${ }^{3}$, Oriel Fajardo de Campos ${ }^{5}$, Dilermando Miranda da Fonseca ${ }^{3}$, Luciana Navajas Rennó 6}

\author{
1 Pesquisa parcialmente financiada pelo CNPq. \\ 2 Programa de Pós-graduação em Zootecnia - UFV, Viçosa, MG. \\ ${ }^{3}$ Departamento de Zootecnia/UFV, Viçosa, MG. Bolsista do CNPq. \\ ${ }^{4}$ Departamento de Economia Rural/UFV, Viçosa, MG. \\ 5 EMBRAPA Gado de Leite, Juiz de Fora, MG. \\ ${ }^{6}$ Curso de Medicina Veterinária/UNIPAC, Juiz de Fora, MG.
}

RESUMO - Objetivou-se aplicar um modelo de simulação para avaliação bioeconômica de estratégias de alimentação para rebanhos leiteiros e avaliar a produtividade física e a eficiência bioeconômica de sistemas de alimentação com diversas estratégias de alimentação à base de volumosos para vacas de cinco níveis de produção de leite. Utilizou-se uma plataforma computacional desenvolvida com os programas CNCPS v.5.0 e planilhas eletrônicas do Microsoft Excell ${ }^{\circledR}$, de forma a simular a produção e as exigências de nutrientes de uma lactação completa para vacas de diferentes níveis de produção. Foram realizadas análises econômicas em sete estratégias de alimentação. A avaliação da receita subtraída dos custos com alimentação (RMCA) comprovou interação entre a estratégia de alimentação e o nível de produção de leite. As estratégias com alimentação à base de silagem de milho durante a época da seca e pastagens na época das águas resultaram em maiores RMCA para todos os níveis de produção de leite, apesar de as demais estratégias apresentarem resultados próximos dependendo do nível de produção de leite. Nas estratégias avaliadas, quanto maior a produção de leite por vaca maior a produtividade (PROD/ha) e a RMCA por área (RMCA/ha). Quanto maior a capacidade de suporte dos volumosos, ou quanto maior a taxa de lotação que determinada área foi submetida, considerando determinada estratégia de alimentação e determinado nível de produção de leite, maior a PROD/ha e RMCA/ha. Para a RMCA por vaca, volumosos de maior densidade energética resultam em diminuição dos custos de alimentação e aumento da receita por animal. A RMCA/ha é fortemente influenciada pela capacidade de suporte das forrageiras em todos os níveis de produção.

Palavras-chave: bovinos de leite, eficiência bioeconômica, simulação

\section{Bioeconomic evaluation of feeding strategies in milk production systems. 1. Production per animal and per area}

\begin{abstract}
This work was carried out to apply a simulation model for the bioeconomic evaluation of feeding strategies for dairy herds and evaluate the physical productivity and the bioeconomic efficiency of feeding systems for dairy cows using feeding forage based strategies for cows with five levels of milk yield. An computational platform developed with the programs CNCPS v5.0 and electronic spreadsheets of Microsoft Excell ${ }^{\circledR}$ was used, in way to simulate the production and demands of nutrients of a complete lactation for cows of different milk yield levels. Economic analyses in seven strategies of feeding were carried out. The income over feed costs (RMCA) showed interaction among the feeding strategy with the milk yield levels. The strategies based on corn silage during the dry season and pastures during rain season resulted in higher RMCA for all milk yield levels, although the other feeding strategies present closed results, depending on the milk yield level. In the evaluated strategies, as higher was the milk yield per cow, greater was the productivity (PROD/ha) and the RMCA per area (RMCA/ha). As higher was the carrying capacity of forages or the stoking rate, which determined area was submitted, considering determined feeding strategy and milk production level, higher was PROD/ha and the RMCA/ha. For RMCA per cow, forages of greater energy density result in decreased feeding costs and increase in the income per animal. The RMCA/ha was strongly influenced by the support capacity of the forages, in all milk yield levels.
\end{abstract}

Key Words: bioeconomic efficiency, dairy cattle, simulation

Este artigo foi recebido em 1/2/2006 e aprovado em 10/10/2007.

Correspondências devem ser enviadas para francisco.renno@usp.br.

* Endereço atual: Departamento de Nutrição e Produção Animal, Faculdade de Medicina Veterinária e Zootecnia da USP, Campus de Pirassununga, SP. 


\section{Introdução}

A intensificação dos sistemas de produção, com a introdução de tecnologias que oferecem condições de alimentação, de manejo e de sanidade mais adequadas, buscando a melhoria da produtividade animal sobre bases econômicas, tem contribuído para o desempenho positivo de toda cadeia produtiva da pecuária, constituindo em estratégia de competitividade para os produtores envolvidos nessas atividades.

A alimentação de animais explorados em sistemas de produção de leite tem grande influência sobre a lucratividade dessas empresas rurais. Conforme citaram Bath \& Sosnik (1992) e Pereira (2000), entre os fatores que influenciam a eficiência de produção em sistemas de pro dução de leite, o custo com a alimentação do rebanho é a mais importante variável dos custos operacionais de produção.

Segundo Ferreira (2002) e Nogueira (2004a), os concentrados representam 30 a $40 \%$ dos custos operacionais totais, sendo, portanto, os de maior importância. Os mesmos autores relatam que, para sistemas de produção de leite de média e alta tecnologia, os gastos com volumosos são de 17,0 a 30,0\% do custo operacional total de produção. Desta forma, considerando os alimentos concentrados, os insumos para a produção de volumosos (fertilizantes, sementes, etc), mão-de-obra, combustível, maquinários e energia elétrica para produzir, cortar, misturar e fornecer os alimentos aos animais, a alimentação do rebanho pode representar até $65 \%$ do custo total de produção do leite (Bath \& Sosnik, 1992; Nogueira, 2004a).

Os índices de produtividade em sistemas de produção com bovinos estão intimamente relacionadosà qualidade e quantidade do volumoso produzido, que, por meio de seus custos de produção e utilização, influenciam a rentabilidade desses sistemas. Ely (1992) relatou que o valor nutritivo das forragens é crítico para o sucesso dos sistemas de produção de leite, portanto, a definição da estratégia de alimentaçãodeve serbaseada na escolha de forragens que supram adequadamente os nutrientes durante um ciclo produtivo anual. A decisão sobre qual forragem será utilizada depende de múltiplos fatores que determinam a escolha daquela forragem para determinado rebanho (Ely, 1992).

Segundo Nussio \& Nussio (2003), programas de alimentação de bovinos leiteiros devem fundamentar suas diretrizes de acordo com as necessidades dos animais em termos de ingestão de nutrientes, qualitativa e quantitativamente, ao longo de uma lactação ou de um período produtivo, considerando as diversas modificações nas exigências nutricionais desses animais nas diferentes fases do ciclo produtivo.

Leite \& Gomes (2001) citaram que a avaliação bioeconômica de alimentos utilizados para a produção de leite se torna cada vez mais importante, uma vez que a maximização de lucros se torna uma necessidade. A escolha da fonte mais adequada de forragem suplementar é uma função multidisciplinar e, desta forma, a utilização de métodos de simulação tem se tornado uma ferramenta ideal para prever o retorno econômico gerado pela intensificação da produção (Nussio \& Nussio, 2003).

Nussio \& Nussio (2003), utilizando as exigências nutricionais e o valor nutritivo dos alimentos estimados pelo NRC (2001), avaliaram a produtividade e geração de receita com a utilização de forrageiras de inverno para vacas de dois níveis de produção e verificaram que o fornecimento de silagem de milho para vacas de 15 e $25 \mathrm{~kg} /$ dia propiciou menor custo de alimentação, principalmente pelo menor uso de concentrados e pela maior utilização dos volumosos nas formulações das rações. No entanto, na avaliação da produtividade e receita por unidade de área, a utilização da cana-de-açúcar apresentou melhores resultados. Esse es tudo foi desenvolvido com base na metodologia de simulação proposta por Undersander et al. (1993), que utiliza equações sucessivas, por meio de planilha eletrônica, na determinação da produtividade e eficiência econômica de estratégias de alimentação.

Objetivou-se com este estudo utilizar uma plataforma computacional para a avaliação bioeconômica de estratégias de alimentação para rebanhos leiteiros e avaliar a produtividade física e a eficiência bioeconômica de sis temas de alimentação para vacas em lactação considerando cinco níveis de produção de leite em sete estratégias de alimentação.

\section{Material e Métodos}

Utilizou-se uma plataforma computacional (Figura 1) que representa a produção de leite de uma lactação completa, desenvolvida utilizando-se o software CNCPS (Cornell Net Carbohydrate and Protein System), versão 5.0 (Fox et al., 2003), para a formulação das dietas. Com os resultados, por meio de planilhas eletrônicas e utilizando-se o software Microsoft EXCELL ${ }^{\circledR}$, foram realizados os cálculos de eficiência bioeconômica dos sistemas de alimentação avaliados.

Nas simulações com o CNCPS, consideraram-se as necessidades nutricionais de uma vaca leiteira com cinco níveis de produção por lactação. Para a oferta de nutrientes, definiram-se o valor nutritivo e as características agronômicas 
das forrageiras utilizadas de acordo com dados da literatura. Modificações nas necessidades dietéticas das vacas com o aumento da produção ou a modificação da base forrageira atribuídas às estratégias de alimentação resultam em alterações no nível de utilização de forragens e de concentrado, alterando a produtividade por área e a eficiência bioeconômica das estratégias de alimentação.

Considerou-se que os animais eram da raça Holandesa, tinham idade adulta de 48 meses, peso vivo médio de $600 \mathrm{~kg}$ após o parto e que ficaram prenhes aos 120 dias pós-parto, portanto, com intervalo de partos de 13,2 meses. Foram considerados oito períodos de lactação para obtenção da produção de leite e das exigências nutricionais e para a formulação das dietas: 14, 42, 70, 112, 168, 231, 294 e 305 dias de lactação. A escolha desses dias em lactação foi baseada na capacidade de representarem adequadamente as necessidades de nutrientes e a dinâmica do ciclo produtivo, características de cada fase da lactação em vacas leiteiras.

Para estimativa da produção média de leite em cada período de lactação, adotou-se o modelo proposto por

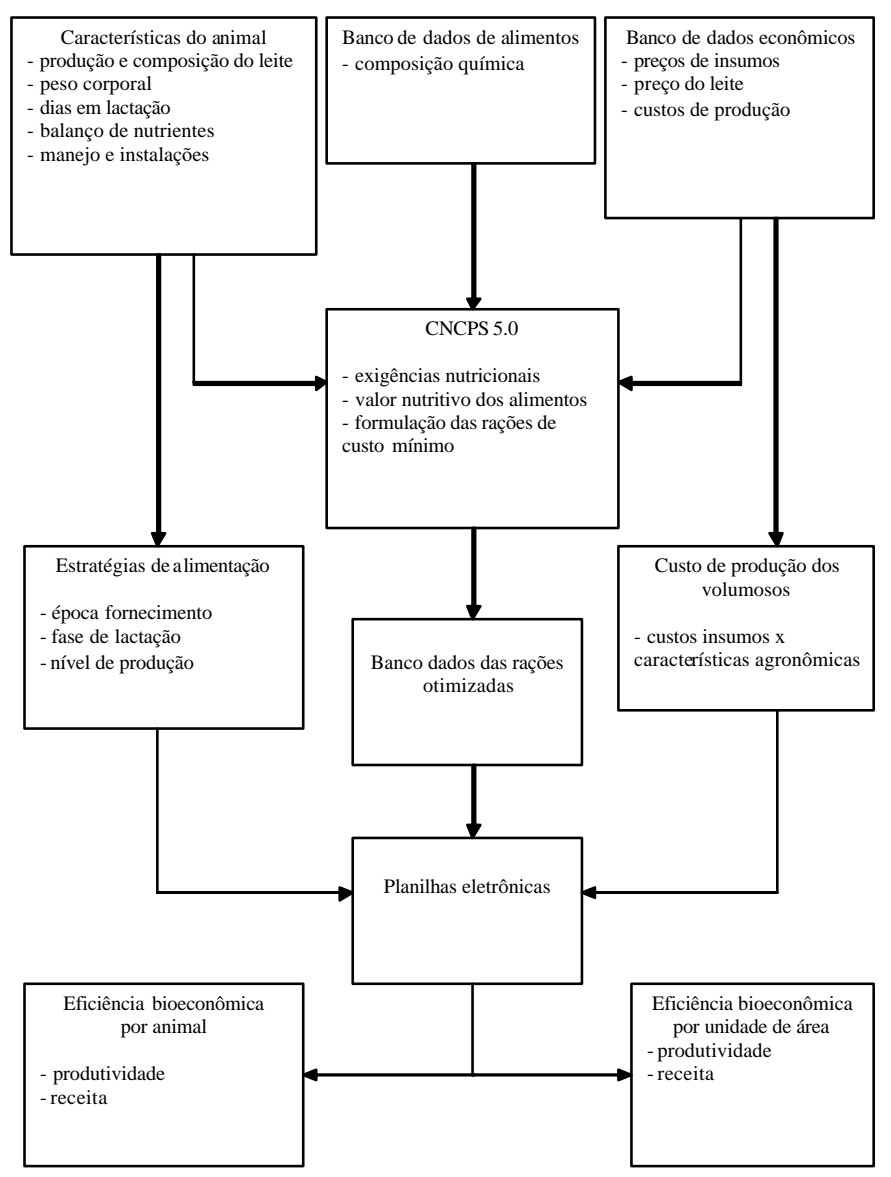

Figura 1 - Diagrama da estrutura computacional utilizada nas simulações.
Congleton \& Everett (1980). Foram avaliados cinco níveis de produção de leite por lactação (kg de leite em 305 dias): 3.075,6; 4.473,7; 6.151,3; 7.549,3; e 9.227,0, denominados, respectivamente, nível de produção baixo (B), médio-baixo (MB), médio (M), médio-alto (MA) e alto (A), que corresponderam à produção média de 10,$1 ; 14,7 ; 20,2 ; 24,8$ e $30,3 \mathrm{~kg} /$ dia em 305 dias de lactação. Considerou-se que as produções de leite apresentaram composição de $3,8 \%$ de gordura e 3,3\% de proteína total.

A produção total de leite na lactação foi calculada utilizando-se as médias das produções de leite estimadas segundo o modelo citado anteriormente. Calculou-se a média entre dois pontos consecutivos multiplicada pelo número de dias do período, obtendo-se, desta forma, as produções parciais ao longo da lactação. A produção final correspondeu ao somatório dessas produções parciais.

No cálculo do potencial de produção, foi considerada a adequada mobilização de reservas corporais. Desse modo, para todas as estratégias de alimentação avaliadas nos níveis de produção de leite, considerou-se que as rações foram suficientes para sustentar a produção de leite e propiciar o retorno à condição corporal inicial quando as vacas estariam no final da lactação. Introduziu-se um limite para a ocorrência de mobilização de reservas corporais no início de lactação até 70 dias pós-parto, de modo que esta mobilização não foi superior a uma unidade (1) da escala de avaliação do escore da condição corporal (ECC) em comparação ao ECC no momento do parto.

Neste estudo, o ECC no momento do parto foi considerado 3,5 , uma vez que a maximização da produção de leite foi obtida com este escore no momento do parto (Waltner et al., 1993). A partir de 70 dias pós-parto, exigiu-se que os animais apresentassem balanço energético e protéico próximo de zero e que, a partir de 112 dias de lactação, fossem fornecidas quantidades de nutrientes superiores às exigências nutricionais para que os animais recuperassem até os 305 dias de lactação as reservas corporais mobilizadas no início da lactação, conforme recomendações de Van Soest (1994), Fox et al. (2003) e NRC (2001).

No cálculo das exigências nutricionais de mantença, o peso corporal variou conforme equação proposta por Rotz et al. (1999) e o peso corporal segundo os dias de lactação. Utilizou-se o software CNCPS, versão 5.0 (Fox et al., 2003), para cálculo das dietas e estimativa das exigências nutricionais e do valor nutritivo dos alimentos. Na estimativa do valor nutritivo dos alimentos, utilizaram-se as equações do Nível 1. Exigiu-se que todas as dietas formuladas atendessem às recomendações de balanço de nutrientes em relação à fase da lactação, independentemente do volumoso ou dos componentes dos concentrados utilizados. 
Para determinação da máxima participação dos volumosos nas dietas, foram atendidas as recomendações de Mertens (1992) para vacas de diferentes períodos de lactação com consumo de FDN de 1,0 a 1,4\% do peso vivo, o que resultou no fornecimento de 100 a $200 \%$ das exigências mínimas de fibra em detergente neutro (FDN) estimadas pelo CNCPS. Também foi considerado que as dietas fornecessem nutrientes para propiciar balanço de nitrogênio e peptídeos no rúmen de no mínimo 100 a $125 \%$, pH ruminal não inferior a 6,2 e suprimento de lisina e metionina de no mínimo $100 \%$.

Em virtude dos elevados níveis de concentrado utilizados em algumas dietas simuladas, adicionou-se bicarbonato de sódio (tamponante), aproximadamente $120 \mathrm{~g} / \mathrm{vaca} / \mathrm{dia}$, nas dietas com mais de $40 \%$ de concentrado na matéria seca. Também foram adicionados minerais e vitaminas aos concentrados, fixando-se $2,5 \%$ de mistura mineral e de premix vitamínico para todas as dietas.

Considerou-se temperatura ambiente de $25^{\circ} \mathrm{C}$ e umidade relativa do ar de $40 \%$. Para as estratégias de alimentação com utilização de animais em pastejo, foi considerado que os animais apresentaram exigências nutricionais adicionais para esta atividade, conforme recomendam Fox et al. (2003) e o NRC (2001), sendo especificado que cada vaca, ao pastejar, caminha $3,0 \mathrm{~km}$ por dia em terreno plano. Quando os alimentos foram fornecidos no cocho, ou seja, que não houve atividade de pastejo,considerou-seque os animais permaneceram em regime de confinamento tipo "free-stall", de até 200 vacas, caminhando $500 \mathrm{~m}$ por dia, segundo Fox et al. (2003).

Para o cálculo das dietas, foi utilizado o otimizador de rações que acompanha o software CNCPS, baseado em programação linear. Formularam-se rações com custo mínimo considerando os diferentes alimentos disponíveis e dos parâmetros nutricionais exigidos.

Como alimentos concentrados foram utilizados componentes dietéticos comumente encontrados no mercado: fubá de milho, farelo de soja, farelo de algodão, farelo de trigo, polpa cítrica e uréia. Como alimentos volumosos foram utilizados: silagem de milho (Zea mays), cana-deaçúcar (Saccharum sp.), capim-elefante (Pennisetum purpureum, cv. Napier), capim-braquiária Braquiaria brizantha sp.) e capim-tifton 85 (Cynodon dactylon, cv. Tifton 85).

Para uso desses alimentos, recorreu-se ao banco de dados de composição de alimentos tropicais desenvolvido por Tedeschi et al. (2002), que acompanha o software CNCPS v.5.0, complementado com informações de composição de alimentos descritas por Valadares Filho et al. (2003).

As variáveis de interesse para interpretação dos resultados, fornecidas pelo CNCPS v.5.0, foram: estimativa da produção de leite de acordo com a dsponibilidade de energia metabolizável (EM) e de proteína metabolizável (PM) (kg/dia); estimativa da mobilização e reposição das reservas corporais a partir da mudança de peso vivo $(\mathrm{kg} /$ dia); estimativa de parâmetros ruminais a partir da PM microbiana (g/dia); balanço de peptídeoe $\mathrm{N}$ ruminal (g/dia); $\mathrm{pH}$ e excesso de $\mathrm{N}$ excretado (g/dia).

Para a avaliação dos volumosos, foram desenvolvidas sete estratégias de alimentação para uma lactação completa, variando entre sistemas que utilizam um único volumoso ao longo do ano a sistemas onde são utilizadas pastagens na época das águas (outubro a março) como volumoso exclusivo, e suplementação com silagem ou cana-de-açúcar na época das secas (abril a setembro): estratégia (EST) 1 silagem de milho (SIM) como volumoso exclusivo ao longo de toda lactação (SIM); EST 2 - SIM durante a época seca e pastejo em capim-braquiária (BRI) na época das águas (SIM+BRI); EST 3 - SIM durante a época seca e pastejo de capim-elefante (NAP) na época das águas (SIM+NAP); EST 4 - SIM durante a época seca e pastejo em capim-tifton 85 (TIF) na época das águas (SIM+TIF); EST 5 - cana-deaçúcar (CAN) durante a época seca e pastejo de BRI na época das águas (CAN+BRI); EST 6-CAN durante a época seca e pastejo de NAP na época das águas (CAN+NAP); EST 7 -CAN durante a época seca e pastejo de TIF na época das águas (CAN+TIF).

Considerou-se o mês de maio, início da época seca, como o mês de parto padrão, com a utilização dos diferentes volumosos que variaram conforme os dias de lactação. Desta forma, aproximadamente até o meio da lactação, assumiu-se que o animal recebeu dieta à base de forrageiras típicas da época seca, ou seja, silagem de milho e cana-deaçúcar. Na segunda metade da lactação, o volumoso padrão foi constituído de pastagens, com exceção da estratégia SIM. A descrição detalhada das rações formuladas, para cada fase da lactação, nível de produção e estratégia de alimentação é apresentada em Rennó (2005).

Considerando as sete estratégias de alimentação avaliadas e a metodologia utilizada nas formulações de rações, com avaliações de dietas em oito pontos na curva de lactação e envolvendo um animal médio com cinco níveis de produção, foram realizadas aproximadamente 375 simu lações no CNCPS v.5.0 para a obtenção dos dados para a avaliação bioeconômica da utilização dos alimentos. Considerando ainda que, nas fases de definição da metodologia utilizada e de ajustes no sistema de formulação de rações adotado, foram simuladas aproximadamente 840 dietas no CNCPS v.5.0.

Nas avaliações bioeconômicas, foram utilizados valores comumente encontrados na literatura para as caracterís ticas 
agronômicas das forragens (Tabela 1). Na determinação dos custos de produção da silagem de milho, foi considerada uma perda de $5 \%$ resultante do processo de ensilagem, e também de $5 \%$ para as perdas resultantes da distribuição e do arraçoamento dos animais. Para a cana-de-açúcar, foi considerado que as perdas resultantes da distribuição e do arraçoamento dos animais foram de $5 \%$.

Para as pastagens avaliadas, considerou-se que $90 \%$ ou mais da produção anual de forragem ocorreu no período das águas, ou seja, de outubro até abril. As pastagens não foram utilizadas como fonte de volumosos na época da seca, em virtude da marcante sazonalidade de produção forrageira (Botrel \& Xavier, 1998). Para avaliação dos custos de produção, foi considerado que durante o processo de pastejo os animais teriam oferta de forragem do dobro do consumo, correspondendo, portanto, a uma eficiência de colheita de forragem de $50 \%$ do disponível. Para todas as forrageiras utilizadas nesse estudo, os cálculos detalhados dos custos de implantação, manutenção ou de produção estão descritos em Rennó (2005).

Os preços do leite e dos insumos utilizados nesse estudo foram obtidos por meio da Scott Consultoria (2003) e se referem ao período de janeiro a dezembro de 2003. Nas análises econômicas, foram usados os preços deflacionados até dezembro de 2003, de modo que os preços mensais foram utilizados para determinação dos custos de produção e da receita de leite de acordo com a variação mensal de preços. No valor dos insumos utilizados, estão embutidos os custos de transporte $(6 \%)$ e impostos $(12 \%)$ e, no preço do leite, estão descontados $3 \%$ de custo de transporte do preço bruto do leite (Scott Consultoria, 2003).

Para o cálculo da quantidade de alimentos fornecida e dos custos com a alimentação ao longo da lactação, foram consideradas as dietas entre dois pontos na curva de lactação, de modoque cada uma fosse fornecida metade do período entre dois pontos sucessivos. Os custos de alimentação foram obtidos considerando as exigências de alimentos para cada período de lactação avaliado e os preços dos insumos naquele período, de acordo com a formulação de ração de custo mínimo realizada pelo CNCPS. O gasto diário com alimentação em determinada fase da lactação foi multiplicado pelo número de dias da fase. Com este procedimento sendo realizado sucessivamente obteve-se a composição dos custos totais de alimentação ao final da lactação.

Posteriormente, foram avaliadas as receitas com a venda do leite, considerando a produção em cada fase da lactação e o preço médio do leite naquele período, com os preços iniciais do leite e dos alimentos relativos ao início da lactação na época seca (maio/2003).

O cálculo da receitas menos os custo de alimentação (RMCA) foi realizado utilizando-se as equações descritas abaixo:

$$
C A T=\left(\Sigma U A_{i j} * D_{j}\right) * R \$ I_{j}
$$

em que CAT é o custo de alimentação total (R\$); $\Sigma \mathrm{UA}$, o somatório da utilização do alimento i ( $\mathrm{kg} / \mathrm{dia})$ no tempo j (dias); D, o número de dias no período j; e R \$I, o custo dos insumos utilizados na alimentação ( $R$ \$) no tempo j (dias).

$$
R B P L=\left(\Sigma P L_{j} * D_{j}\right) * R \$ L_{j}
$$

em que RBPL é a renda bruta com a produção de leite ( $\mathrm{R} \$)$; $\Sigma$ PL, o somatório da produção de leite $(\mathrm{kg})$ no tempo j (dias); D, o número de dias no período j; e R $\$$ L, o preço do leite $(\mathrm{R} \$)$ no tempo $\mathrm{j}$ (dias).

$$
R M C A_{l m}=R B P L_{l m}-C A T_{l m}
$$

em que RMCA = receita menos os custos com alimentação $(\mathrm{R} \$)$ da estratégia de alimentação 1 no nível de produção m; $\mathrm{RBPL}=$ renda bruta da produção de leite $(\mathrm{R} \$)$ da estratégia de alimentação 1 no nível de produção $\mathrm{m}$; e CAT = custos totais de alimentação $(\mathrm{R} \$)$ da estratégia de alimentação 1 no nível de produção $\mathrm{m}$.

Os custos de alimentação foram avaliados por meio das seguintes variáveis: consumo total de concentrados (CTC);

\begin{tabular}{|c|c|c|c|c|c|c|}
\hline Forrageira & MV/ha (t) & MS/ha (t) & $\mathrm{PE}(\%)$ & PA $(\%)$ & $\mathrm{R} \$ / \mathrm{t} . \mathrm{MV}$ & $\mathrm{R} \$ / \mathrm{t} \mathrm{MS}$ \\
\hline Silagem de milho ${ }^{1}$ & 45,0 & 14,1 & 5 & 5 & 54,5 & 173,7 \\
\hline Cana-de-açúcar ${ }^{2}$ & 100,0 & 29,7 & - & 5 & 25,1 & 84,5 \\
\hline Capim-braquiária ${ }^{3}$ & 165,5 & 24,0 & - & 50 & 10,4 & 71,6 \\
\hline Capim-elefante ${ }^{3}$ & 222,2 & 40,0 & - & 50 & 14,9 & 82,8 \\
\hline Capim-tifton $85^{4}$ & 124,2 & 22,2 & - & 50 & 18,3 & 102,1 \\
\hline
\end{tabular}

Tabela 1 - Produção de matéria verde (MV/ha) e matéria seca (MS/ha) por área, perdas na ensilagem (PE) e durante o arraçoamento (PA) e custos de produção das forragens, avaliados em matéria verde (R\$/t MV) e em matéria seca (R\$/t MS)

${ }^{1}$ Boletim do leite (2002); ${ }^{2}$ Galan et al. (1997); ${ }^{3}$ Silva et al. (1996) e Galan et al. (1997); ${ }^{4}$ Rodrigues et al. (1997) e Cóser et al. (1998). 
relação $\mathrm{kg}$ leite produzidos por $\mathrm{kg}$ de concentrado fornecido (L:C); custo total dos concentrados (CUTC); custo médio por kg de concentrado (CMC); custo total dos volumosos (CTV) e custo total da dieta (CTD). Considerando os custos dos concentrados e as quantidades administradas por vaca/dia, para cada estratégia de alimentação e nível de produção, também foi estimado o custo de concentrado por litro de leite produzido (CMC/L).

As estratégias de alimentação foram analisadas por meio das seguintes variáveis: receita menos o custo de alimentação por lactação (RMCA); produtividade por hectare (PROD/ha); animais por hectare (vaca/ha) e lucratividade por hectare (LUCR/ha). Todas as avaliações realizadas nas estratégias de avaliação consideraram nos resultados um rebanho teórico com $100 \%$ de vacas em lactação.

\section{Resultados e Discussão}

O consumo (CTC) e o custo total do concentrado (CUTC) aumentaram de acordo com o nível de produção em todas as estratégias de alimentação (Tabela 2). Maior produção de leite por lactação se relacionou a maior fornecimento de concentrado por vaca, para todas as estratégias de alimentação avaliadas.

Entre as estratégias de alimentação, a utilização da silagem de milho (SIM) durante toda a lactação resultou em menor uso de concentrado por vaca e, conseqüentemente, em menor custo de concentrados por lactação. Também nas estratégias de alimentação onde foram utilizadas combinações de silagem de milho e pastagens (SIM+BRI, SIM+NAP e SIM+TIF), foram observados menores níveis de utilização e menor custo com concentrados quando comparadas às estratégias de alimentação baseadas em cana-de-açúcar e pastagens (CAN+BRI, CAN+NAP e CAN+TIF) (Tabela 2).

Em termos médios, as estratégias SIM+BRI, SIM+NAP e SIM+TIF utilizaram $21,74 \%$ e as estratégias CAN+BRI, $\mathrm{CAN}+\mathrm{NAP}$ e CAN+TIF $43,42 \%$ a mais de concentrado que a estratégia SIM. Os custos de concentrados nas estratégias de alimentação silagem e pastagens foram semelhantes e $9,44 \%$ acima do custo da estratégia SIM, enquanto nas estratégias de cana-de-açúcar e pastagens gastou-se 38,98\% a mais de concentrados que na estratégia SIM. As estratégias com cana-de-açúcar e pastagens utilizaram 17,80\% e gastaram $26,99 \%$ a mais com concentrados que as estratégias com silagem de milho e pastagens.

A relação L:C (litros de leite por kg de concentrado) diferiram quando são comparados os valores obtidos nos diferentes níveis de produção (Tabela 2). Nos níveis de produção B e MB, foram observadas as melhores eficiências de utilização do concentrado em comparação aos níveis $\mathbf{M}$, MA e A. Esses resultados são justificados pela menor necessidade de fornecer concentrados às vacas de menor produção em comparação às de maior produção. As estratégias de alimentação à base de silagem de milho associada ou não às pastagens apresentaram as melhores eficiências de utilização de concentrados em comparação às dietas à base de cana-de-açúcar e pastagens (Tabela 2).

Os custos médios por $\mathrm{kg}$ de concentrado (CMC) foram maiores para as dietas das vacas de menor produção em todas as estratégias avaliadas (Tabela 2), com exceção da estratégia de alimentação CAN+TIF, em razão da composição bromatológica mais equilibrada, especialmente em relação ao teor de proteína bruta da pastagem. Dessa forma, a necessidade de maior percentual de fontes protéicas nos concentrados das vacas de menor produção resultou em maiores CMC, principalmente nos dois menores níveis de produção (B e MB). No entanto, em virtude da composição bromatológica dos volumosos, as estratégias à base de silagem de milho e pastagens apresentaram os menores CMC de concentrado, principalmente as combinações de SIM+NAP e SIM+TIF.

As estratégias de alimentação com cana-de-açúcar apresentaram resultados intermediários, e as estratégias com silagem de milho como volumoso exclusivo, bem como SIM+BRI e CAN+BRI, apresentaram os maiores custos por $\mathrm{kg}$ de concentrado. Estes resultados são decorrentes da maior participação de fontes protéicas nos concentrados, que são de custo mais elevado em relação aos demais componentes, o que resulta em maior custo total do concentrado nestas estratégias de alimentação.

$\mathrm{Na}$ avaliação do CMC/L, as estratégias com silagem de milho apresentaram os menores gastos com concentrados, com médias de $\mathrm{R} \$ 0,164, \mathrm{R} \$ 0,179, \mathrm{R} \$ 0,164$ e $\mathrm{R} \$ 0,159$ por litro de leite produzido para as estratégias SIM, SIM+BRI, SIM+NAP e SIM+TIF, respectivamente. As estratégias com alimentação à base de cana-de-açúcar e pastagens apresentaram médias de $\mathrm{R} \$ 0,224, \mathrm{R} \$ 0,209$ e R \$ 0,210 por litro de leite produzido, respectivamente, para as estratégias CAN+BRI, CAN+NAP e CAN+TIF. Na média, as estratégias baseadas em silagem de milho tiveram custo médio de concentrado por litro de leite produzido cinco centavos menor que o das estratégias à base de cana-de-açúcar.

Apesar de algumas estratégias de alimentação apresentarem maior custo por $\mathrm{kg}$ de concentrado, as estratégias que apresentaram estes resultados propiciaram os menores custos com concentrados por litro de leite produzido. De forma semelhante, quando avaliados os custos por litro de leite produzido em relação aos diferentes níveis de produção, observou-se que, mesmo com os maiores custos por kg de 
Tabela 2 - Consumo total de concentrados (CTC), relação kg leite produzidos por kg de concentrado fornecido (L:C), custo total dos concentrados (CUTC), custo médio por kg de concentrado (CMC), custo do concentrado por litro de leite produzido (CMC/ $\mathrm{L}$ ), custo total dos volumosos (CTV) e custo total da dieta (CTD) em cada estratégia de alimentação para vacas de diferentes níveis de produção de leite por lactação (305 dias)

\begin{tabular}{|c|c|c|c|c|c|c|c|}
\hline \multirow{2}{*}{$\begin{array}{l}\text { Estratégia de } \\
\text { alimentação }\end{array}$} & \multirow[t]{2}{*}{ Variável } & \multirow[t]{2}{*}{ Unidade } & \multicolumn{5}{|c|}{ Nível de produção } \\
\hline & & & Baixo & Médio-baixo & Médio & Médio-alto & Alto \\
\hline \multirow[t]{7}{*}{ SIM } & $\mathrm{CTC}$ & $\mathrm{kg}$ & 836,31 & $1.092,34$ & $1.812,28$ & $2.198,56$ & $3.037,26$ \\
\hline & $\mathrm{L}: \mathrm{C}$ & $\mathrm{L}: \mathrm{kg}$ & 3,68 & 4,10 & 3,39 & 3,43 & 3,04 \\
\hline & CUTC & $\mathrm{R} \$$ & 526,83 & 672,95 & 987,11 & $1.204,65$ & $1.656,69$ \\
\hline & $\mathrm{CMC}$ & $\mathrm{R} \$$ & 0,630 & 0,616 & 0,545 & 0,548 & 0,545 \\
\hline & $\mathrm{CMC} / \mathrm{L}$ & $\mathrm{R} \$$ & 0,180 & 0,160 & 0,160 & 0,150 & 0,171 \\
\hline & CTV & $\mathrm{R} \$$ & 493,89 & 565,97 & 556,33 & 566,33 & 579,79 \\
\hline & CTD & $\mathrm{R} \$$ & $1.020,72$ & $1.238,92$ & $1.543,44$ & $1.770,99$ & $2.176,48$ \\
\hline \multirow[t]{7}{*}{ SIM+BRI } & $\mathrm{CTC}$ & $\mathrm{kg}$ & 730,56 & $1.336,89$ & $2.297,36$ & $2.809,50$ & - \\
\hline & $\mathrm{L}: \mathrm{C}$ & $\mathrm{L}: \mathrm{kg}$ & 4,21 & 3,35 & 2,68 & 2,69 & - \\
\hline & CUTC & $\mathrm{R} \$$ & 472,21 & 768,41 & $1.201,57$ & $1.472,12$ & - \\
\hline & $\mathrm{CMC}$ & $\mathrm{R} \$$ & 0,646 & 0,575 & 0,523 & 0,524 & - \\
\hline & $\mathrm{CMC} / \mathrm{L}$ & $\mathrm{R} \$$ & 0,195 & 0,195 & 0,172 & 0,154 & - \\
\hline & CTV & $\mathrm{R} \$$ & 359,64 & 338,18 & 341,28 & 338,55 & - \\
\hline & CTD & $\mathrm{R} \$$ & 831,84 & $1.106,59$ & $1.542,84$ & $1.810,67$ & - \\
\hline \multirow[t]{7}{*}{ SIM+NAP } & $\mathrm{CTC}$ & $\mathrm{kg}$ & 723,85 & $1.395,50$ & $2.350,86$ & $2.858,74$ & - \\
\hline & $\mathrm{L}: \mathrm{C}$ & $\mathrm{L}: \mathrm{kg}$ & 4,25 & 3,21 & 2,62 & 2,64 & - \\
\hline & CUTC & $\mathrm{R} \$$ & 388,93 & 694,78 & $1.142,89$ & $1.418,08$ & - \\
\hline & CMC & $\mathrm{R} \$$ & 0,537 & 0,498 & 0,486 & 0,496 & - \\
\hline & $\mathrm{CMC} / \mathrm{L}$ & $\mathrm{R} \$$ & 0,188 & 0,186 & 0,155 & 0,126 & - \\
\hline & CTV & $\mathrm{R} \$$ & 359,64 & 338,18 & 341,28 & 338,55 & - \\
\hline & CTD & $\mathrm{R} \$$ & 770,43 & $1.037,05$ & $1.489,62$ & $1.760,72$ & - \\
\hline \multirow[t]{7}{*}{ SIM+TIF } & $\mathrm{CTC}$ & $\mathrm{kg}$ & 707,99 & $1.289,20$ & $2.299,02$ & $2.893,98$ & - \\
\hline & $\mathrm{L}: \mathrm{C}$ & $\mathrm{L}: \mathrm{kg}$ & 4,34 & 3,47 & 2,68 & 2,61 & - \\
\hline & CUTC & $\mathrm{R} \$$ & 362,32 & 653,16 & $1.128,06$ & $1.432,57$ & - \\
\hline & $\mathrm{CMC}$ & $\mathrm{R} \$$ & 0,512 & 0,507 & 0,491 & 0,495 & - \\
\hline & CMC/L & $\mathrm{R} \$$ & 0,190 & 0,183 & 0,146 & 0,118 & - \\
\hline & CTV & $\mathrm{R} \$$ & 422,72 & 366,12 & 378,53 & 366,48 & - \\
\hline & CTD & $\mathrm{R} \$$ & 785,04 & $1.019,28$ & $1.506,60$ & $1.799,05$ & - \\
\hline \multirow[t]{7}{*}{$\mathrm{CAN+BRI}$} & CTC & $\mathrm{kg}$ & $1.015,92$ & $1.633,02$ & $2.589,89$ & $3.205,28$ & - \\
\hline & $\mathrm{L}: \mathrm{C}$ & $\mathrm{L}: \mathrm{Kg}$ & 3,03 & 2,74 & 2,38 & 2,36 & - \\
\hline & CUTC & $\mathrm{R} \$$ & 616,62 & 983,02 & $1.456,05$ & $1.819,19$ & - \\
\hline & $\mathrm{CMC}$ & $\mathrm{R} \$$ & 0,607 & 0,602 & 0,562 & 0,568 & - \\
\hline & $\mathrm{CMC} / \mathrm{L}$ & $\mathrm{R} \$$ & 0,241 & 0,237 & 0,220 & 0,200 & - \\
\hline & CTV & $\mathrm{R} \$$ & 233,77 & 183,72 & 192,93 & 184,15 & - \\
\hline & CTD & $\mathrm{R} \$$ & 850,40 & $1.166,75$ & $1.648,98$ & $2.003,33$ & - \\
\hline \multirow[t]{7}{*}{$\mathrm{CAN}+\mathrm{NAP}$} & $\mathrm{CTC}$ & $\mathrm{kg}$ & 945,99 & $1.693,36$ & $2.691,32$ & $3.230,56$ & - \\
\hline & $\mathrm{L}: \mathrm{C}$ & $\mathrm{L}: \mathrm{kg}$ & 3,25 & 2,64 & 2,29 & 2,34 & - \\
\hline & CUTC & $\mathrm{R} \$$ & 526,02 & 909,36 & $1.409,65$ & $1.768,60$ & - \\
\hline & $\mathrm{CMC}$ & $\mathrm{R} \$$ & 0,556 & 0,537 & 0,524 & 0,547 & - \\
\hline & CMC/L & $\mathrm{R} \$$ & 0,234 & 0,229 & 0,203 & 0,171 & - \\
\hline & CTV & $\mathrm{R} \$$ & 259,04 & 195,9 & 203,21 & 196,33 & - \\
\hline & CTD & $\mathrm{R} \$$ & 785,06 & $1.105,21$ & $1.612,87$ & $1.964,93$ & - \\
\hline \multirow[t]{7}{*}{ CAN+TIF } & CTC & $\mathrm{kg}$ & $1.025,95$ & $1.637,52$ & $2.630,70$ & $3.255,51$ & - \\
\hline & $\mathrm{L}: \mathrm{C}$ & $\mathrm{L}: \mathrm{kg}$ & 3,00 & 2,73 & 2,34 & 2,32 & - \\
\hline & CUTC & $\mathrm{R} \$$ & 525,35 & 881,97 & $1.421,36$ & $1.823,63$ & - \\
\hline & $\mathrm{CMC}$ & $\mathrm{R} \$$ & 0,512 & 0,539 & 0,540 & 0,560 & - \\
\hline & CMC/L & $\mathrm{R} \$$ & 0,242 & 0,231 & 0,197 & 0,171 & - \\
\hline & CTV & $\mathrm{R} \$$ & 289,09 & 219,42 & 235,87 & 219,85 & - \\
\hline & CTD & $\mathrm{R} \$$ & 814,45 & $1.101,40$ & $1.657,22$ & $2.043,48$ & - \\
\hline
\end{tabular}

$\mathrm{SIM}$ = silagem de milho como volumoso exclusivo; $\mathrm{SIM}+\mathrm{BR}$ = silagem de milho durante a época seca e pastejo em capim-braquiária durante a época das águas; SIM+NAP = silagem de milho durante a época seca e pastejo de capim-elefante durante a época das águas; SIM+TIF = silagem de milho durante a época seca e pastejo em capim-tifton 85 durante a época das águas; CAN+BRI = cana-de-açúcar durante a época seca e pastejo em capim-braquiária durante a época das águas; CAN+NAP = cana-de-açúcar durante a época seca e pastejo de capim-elefante durante a época das águas; CAN+TIF = cana-de-açúcar durante a época seca e pastejo em capim-tifton 85 durante a época das águas. 
concentrado, os níveis de menor produção apresentaram os menores gastos de concentrado por litro de leite produzido nas diferentes estratégias de alimentação avaliadas.

Esses resultados comprovam que avaliações do uso de concentrados para vacas em lactação baseadas somente no custo por $\mathrm{kg}$ de concentrado devem ser realizadas com cautela, pois podem não representar economicamente qualquer tipo de vantagem em relação aos custos de alimentação por litro de leite produzido, seja em diferentes estratégias de alimentação, e para vacas de variados níveis de produção, como as avaliadas neste estudo.

Entre os níveis de produção, com exceção do nível B, que apresentou maior gasto com volumosos em decorrência de sua maior participação nas dietas, para os demais níveis de produção os custos, foram semelhantes dentro da mesma estratégia de alimentação. No entanto, quando avaliados os custos totais com volumosos (CTV) entre as estratégias de alimentação, estes são diferentes (Tabela 2).

Quanto maior a participação da silagem de milho nas dietas, maiores os CTV. A estratégia SIM apresentou os maiores gastos com volumosos. Em um nível de gasto intermediário estão posicionadas as estratégias SIM+TIF, que tiveram maior custo de volumoso em relação àquelas com utilização de silagem (SIM+BRI e SIM+NAP). As estratégias de alimentação à base de cana-de-açúcar pos sibilitaram os menores CTV. Na média, a estratégia SIM apresentou CTV 150,54\% maior que as estratégias à base de cana-de-açúcar e $51,40 \%$ maior que as demais estratégias com utilização silagem de milho, demonstrando que maior participação da silagem de milho nas dietas resulta em maior participação dos volumosos nos custos de alimentação.

$\mathrm{Na}$ avaliação dos custos com alimentação (CTD), são avaliados a combinação dos custos de concentrados e dos volumosos (Tabela 2). Quanto maior o nível de produção de leite, maiores as exigências de nutrientes e maiores os CTD. Os custos com alimentação são proporcionais ao volume total de leite produzido. Nessa avaliação houve diferença nos resultados em relação ao nível de produção.

No nível $\mathrm{B}$ e $\mathrm{MB}$, as estratégias à base de silagem de milho e pastagens (especificamente SIM+NAP e SIM+TIF) e as estratégias com cana-de-açúcar (CAN+NAP e CAN+TIF) apresentaram os menores custos de alimentação. Em posição intermediária ficaram as estratégias SIM+BRI e CAN+BRI. Nesses níveis de produção, a utilização da silagem de milho como volumoso exclusivo (SIM) resultou nos maiores custos de alimentação (CTD). Em porcentagem, a estratégia SIM apresentou custos de alimentação de 28,26 e 24,99\% no nível B e de 17,51 e 10,18\% no nível MB, superiores, na média, às estratégias à base de silagem de milho e pastagens, e cana-de-açúcar e pastagens, respectivamente.

No nível M, as estratégias de alimentação SIM+NAP e SIM+TIF promoveram os melhores resultados, seguidas das estratégias SIM e SIM+BRI. Na média, as estratégias com silagem e pastagens apresentaram custos de alimentação somente 2,01\% inferiores à SIM. As estratégias $\mathrm{CAN}+\mathrm{BRI}, \mathrm{CAN}+\mathrm{NAP}$ e CAN+TIF, na média, tiveram custos de alimentação $8,37 \%$ superiores aos das estratégias com silagem de milho e pastagens. No nível MA, novamente as estratégias à base de silagem de milho proporcionaram os melhores resultados. A estratégia SIM apresentou CTD somente $1,02 \%$ melhor que o das estratégias SIM+BRI, SIM+NAP e SIM+TIF. No entanto, entre as estratégias com cana-de-açúcar, a SIM resultou em CTD 13,15\% inferiores à média das demais.

A RMCA possibilitou resultados que acompanham os custos de alimentação (Tabela 3). Quanto menores os custos com alimentação, maior a geração de receita por vaca no mesmo nível de produção, uma vez que a renda bruta obtida com a venda de leite é a mesma. Os resultados obtidos demonstraram interação entre a melhor estratégia de alimentação e o nível de produção. A combinação de silagem de milho durante a época da seca (início de lactação) com pastagens na época das águas (meio e final de lactação) parece ser a opção mais estável entre os níveis de produção avaliados. Em todos os níveis avaliados, estas alternativas apresentaram menores custos de alimentação e maior geração de receita por vaca (RMCA) (Tabela 3 ).

A escolha das estratégias à base de cana-de-açúcar como forrageira no inverno e pastagens no verão, como opção de alimentos volumosos, também depende do nível de produção dos rebanhos. Nos casos de rebanhos de produção média por vaca entre 3.000 e 4.500 kg leite/vaca/lactação, as estratégias que combinem cana-de-açúcar e pastagens podem ser as melhores opções onde não seja possível a utilização das estratégias à base de silagem de milho.

Nesses níveis de produção, a utilização da silagem de milho como volumoso exclusivo não foi a melhor opção quando avaliado o custo de alimentação. Entretanto, para rebanhos de potencial de produção de 6.000 a $7.500 \mathrm{~kg}$ leite/ vaca/lactação, esta estratégia de alimentação apresentou as mesmas vantagens que a utilização da combinação de silagem de milho e pastagens, com reduzidos custos de alimentação e maior receita por vaca (RMCA) (Tabela 3). A utilização de silagem de milho como volumoso exclusivo pode apresentar vantagens de requerer menor suplementação com concentrados durante a lactação, quando se tem restrição de capital ou quando existe dificuldade para a aquisição deste insumo. 
Com exceção da estratégia à base exclusivamente de silagem de milho, quanto maior a produção por vaca, maior a taxa de lotação que pode ser utilizada nas diferentes estratégias de alimentação avaliadas (Tabela 3). O maior nível de produção demanda maior quantidade de concentrados (Tabela 2) e diminui, conseqüentemente, a participação dos volumosos nas dietas. Como existem diferenças na utilização de volumosos entre os níveis de produção, a maior utilização de concentrados por vaca e por unidade de área dentro da mesma estratégia de alimentação propicia maior taxa de lotação nos níveis de produção mais elevados. A estratégia SIM, que foi exceção, não apresentou diferenças nas taxas de lotação entre os níveis de produção, provavelmente em razão de suas desejáveis características nutricionais, que propiciaram maior proporção de volumosos nas dietas, independentemente do nível de produção.

As estratégias de alimentação que utilizaram forrageiras de alta produtividade por unidade de área, como a cana-deaçúcar e o capim-elefante, propiciaram as maiores taxas de lotação em todos os níveis de produção e estratégias de alimentação. Aliada à grande produtividade por área destas forrageiras, também deve ser considerada a maior utilização de concentrados por unidade de área quando utilizadas estratégias de alimentação com estas forrageiras, favorecendo a manutenção de altas taxas de lotação. Utilizando a estratégia SIM como referência, na média, as estratégias SIM+BRI, SIM+NAP, SIM+TIF, CAN+BRI, CAN+NAP e CAN+TIF apresentaram taxa de lotação 1,$76 ; 32,68 ;-2,62$;

Tabela 3 - Receita menos o custo de alimentação por lactação (RMCA), produtividade por hectare (PROD/ha), animais por hectare (vaca/ha) e lucratividade por hectare (LUCR/ha), para vacas de diferentes níveis de produção de leite por lactação, submetidas a diferentes estratégias de alimentação

\begin{tabular}{|c|c|c|c|c|c|c|c|}
\hline \multirow{2}{*}{$\begin{array}{l}\text { Estratégia de } \\
\text { alimentação }\end{array}$} & \multirow[t]{2}{*}{ Variável } & \multirow[t]{2}{*}{ Unidade } & \multicolumn{5}{|c|}{ Nível de produção } \\
\hline & & & Baixo & Médio-baixo & Médio & Médio-alto & Alto \\
\hline \multirow[t]{4}{*}{ SIM } & RMCA & $\mathrm{R} \$$ & 468,40 & 927,09 & $1.434,82$ & $1.884,15$ & $2.290,90$ \\
\hline & PROD/ha & $\mathrm{L} / \mathrm{ha}$ & $13.884,2$ & $1.7750,5$ & $2.4652,1$ & $29.720,2$ & $39.577,0$ \\
\hline & Vaca/ha & Vaca/ha & 4,51 & 3,97 & 4,01 & 3,94 & 4,29 \\
\hline & LUCR/ha & $\mathrm{R} \$$ & $2.112,5$ & 3680,5 & $5.753,6$ & $7.423,6$ & $9.828,0$ \\
\hline \multirow[t]{4}{*}{ SIM+BRI } & RMCA & $\mathrm{R} \$$ & 657,29 & $1.059,42$ & $1.435,41$ & $1.844,46$ & - \\
\hline & PROD/ha & $\mathrm{L} / \mathrm{ha}$ & $11.643,6$ & $17.496,4$ & $27.436,3$ & $34.388,0$ & - \\
\hline & Vaca/ha & Vaca/ha & 3,79 & 3,91 & 4,46 & 4,56 & - \\
\hline & LUCR/ha & $\mathrm{R} \$$ & $2.491,1$ & 4142,3 & $6.401,9$ & $8.410,7$ & - \\
\hline \multirow[t]{4}{*}{ SIM+NAP } & RMCA & $\mathrm{R} \$$ & 718,70 & $1.128,96$ & $1.488,64$ & $1.894,41$ & - \\
\hline & PROD/ha & $\mathrm{L} / \mathrm{ha}$ & $15.582,2$ & $23.011,0$ & $35.400,6$ & $4.4091,2$ & - \\
\hline & Vaca/ha & Vaca/ha & 5,07 & 5,14 & 5,75 & 5,84 & - \\
\hline & LUCR/ha & $\mathrm{R} \$$ & $3.643,8$ & 5802,9 & $8.559,7$ & $1.1063,4$ & - \\
\hline \multirow[t]{4}{*}{ SIM+TIF } & RMCA & $\mathrm{R} \$$ & 704,09 & $1.146,73$ & $1.471,66$ & $1.856,09$ & - \\
\hline & PROD/ha & L/ha & $10.985,0$ & $16.478,1$ & $26.296,4$ & $33.833,2$ & - \\
\hline & Vaca/ha & Vaca/ha & 3,57 & 3,68 & 4,27 & 4,48 & - \\
\hline & LUCR/ha & $\mathrm{R} \$$ & $2.513,6$ & $4.220,0$ & $6.284,0$ & $8.315,3$ & - \\
\hline \multirow[t]{4}{*}{ CAN+BRI } & RMCA & $\mathrm{R} \$$ & 638,73 & 999,25 & $1.329,28$ & $1.651,80$ & - \\
\hline & PROD/ha & L/ha & $15.467,5$ & $23.948,7$ & $38.891,5$ & $50.931,9$ & - \\
\hline & Vaca/ha & Vaca/ha & 5,03 & 5,35 & 6,32 & 6,75 & - \\
\hline & LUCR/ha & $\mathrm{R} \$$ & $3.212,8$ & $5.346,0$ & $8.401,0$ & $1.1149,7$ & - \\
\hline \multirow[t]{4}{*}{$\mathrm{CAN}+\mathrm{NAP}$} & RMCA & $\mathrm{R} \$$ & 704,06 & $1.060,80$ & $1.365,39$ & $1.690,21$ & - \\
\hline & PROD/ha & $\mathrm{L} / \mathrm{ha}$ & $22.163,4$ & $35.683,9$ & $57.691,3$ & $73.648,8$ & - \\
\hline & Vaca/ha & Vaca/ha & 7,21 & 7,98 & 9,38 & 9,76 & - \\
\hline & LUCR/ha & $\mathrm{R} \$$ & $5.076,3$ & $8.465,2$ & $12.807,4$ & $16.496,4$ & - \\
\hline \multirow[t]{4}{*}{$\mathrm{CAN}+\mathrm{TIF}$} & RMCA & $\mathrm{R} \$$ & 674,68 & $1.064,61$ & $1.321,03$ & $1.611,65$ & - \\
\hline & PROD/ha & L/ha & $14.825,6$ & $21.958,0$ & $36.768,2$ & $49.101,2$ & - \\
\hline & Vaca/ha & Vaca/ha & 4,82 & 4,91 & 5,98 & 6,50 & - \\
\hline & LUCR/ha & $\mathrm{R} \$$ & $3.252,0$ & $5.227,2$ & $7.899,8$ & $10.475,7$ & - \\
\hline
\end{tabular}

$\mathrm{SIM}=$ silagem de milho como volumoso exclusivo; $\mathrm{SIM}+\mathrm{BRI}=$ silagem de milho durante a época seca e pastejo em capim-braquiária durante a época das águas; SIM+NAP = silagem de milho durante a época seca e pastejo de capim-elefante durante a época das águas; SIM+TIF = silagem de milho durante a época seca e pastejo em capim-tifton 85 durante a época das águas; CAN+BRI = cana-de-açúcar durante a época seca e pastejo em capim-braquiária durante a época das águas; $\mathrm{CAN}+\mathrm{NAP}=$ cana-de-açúcar durante a época seca e pastejo de capim-elefante durante a época das águas; CAN+TIF = cana-de-açúcar durante a época seca e pastejo em capim-tifton 85 durante a época das águas. 
42,$72 ; 108,95$ e $35,18 \%$ maiores, respectivamente, que a utilização da estratégia SIM.

Em todas as estratégias de alimentação avaliadas, quanto maior a produção por vaca, maior a produtividade por unidade de área (PROD/ha) (Tabela 3). De forma semelhante, a LUCR/ha foi 65,$38 ; 151,58$ e 228,82\% maiores, respectivamente, nos níveis MB, M e MA em comparação ao nível B de produção (Tabela 3). Quanto maior a produção de leite por vaca, maior a receita menos os custos de alimentação por vaca e por unidade de área.

Em relação à produtividade por hectare, quanto maior a capacidade de suporte da estratégia de alimentação adotada, ou, quanto maior a taxa de lotação a que determinada área for submetida dentro de determinado nível de produção, maior poderá ser a produtividade e a receita por unidade de área.

Utilizando-se a estratégia SIM como referência, na média, as PROD/ha das estratégias de alimentação SIM+BRI, SIM+NAP, SIM+TIF, CAN+BRI, CAN+NAP e CAN+TIF foram superiores em 5,76; 37,30; 1,84; 50,27; 119,97 e 42,61\%, respectivamente (Tabela 3). Esta mesma comparação utilizando as estimativas de LUCR/ha comprova que as estratégias SIM+BRI, SIM+NAP, SIM+TIF, CAN+BRI, CAN+NAP e CAN+TIF foram 13,$05 ; 53,24 ; 12,45 ; 48,18$; 125,85 e 41,56\% superiores a estratégia SIM (Tabela 3). Em todos os níveis de produção, a utilização da silagem de milho durante toda a lactação (SIM) resultou em menor receita por unidade de área, enquanto a estratégia de alimentação CAN+NAP apresentou os melhores resultados.

A RMCA por animal é muito influenciada pelo valor nutritivo das forrageiras que resultam em diferentes níveis de utilização de concentrado e, conseqüentemente, em diferentes custos de alimentação por vaca. Paralelamente, existe uma interação entre a melhor opção forrageira e o nível de produção dos animais a serem alimentados. Conforme citaram Nussio et al. (2001), a análise dos dados também revelou forte tendência de interação volumoso $x$ produção individual diária de leite, ou seja, a ordem de classificação dos volumosos preferenciais é específica para determinado nível de produção do animal.

Avaliando os resultados da RMCA observa-se que existe a possibilidade de obtenção de produtividades semelhantes entre algumas fontes de volumosos e estratégias de alimentação para determinado nível de produção, o que, segundo Nussio et al. (2001), cria flexibilidade de opção por culturas agrícolas sob maior diversidade edafoclimática e características diferenciadas das bases físicas dos sistemas de produção de leite.

Comparando os resultados desta pesquisa aos de outros estudos de simulação para avaliar o potencial de utilização de forrageiras, verificam-se tendências semelhantes. Nussio et al. (1998), simulando dados a partir de recomendações nutricionais do NRC (1988), e Nussio \& Nussio (2003), utilizando as exigências nutricionais e o valor nutritivo dos alimentos estimados no NRC (2001), verificaram que a utilização da silagem de milho para vacas de 15 e $25 \mathrm{~kg} /$ dia propicia menor custo de alimentação, principalmente pela menor utilização de concentrados e maior utilização dos volumosos nas formulações das rações.

Desta forma, analisando a produtividade por animal, se o objetivo for a redução dos custos de produção por litro de leite ou aumentar a geração de receita diária por vaca, volumosos de maior densidade energética são mais efetivos em reduzir custos de produção. No entanto, a receita por hectare é fortemente influenciada pela capacidade de suporte das forrageiras em todos os níveis de produção. Mesmo que determinada estratégia de alimentação apresente menores custos de alimentação e, portanto, maior receita por vaca, a taxa de lotação que esta estratégia pode proporcionar altera marcadamente a receita por área.

Nos estudos de Nussio et al (1998) e Nussio \& Nussio (2003), por meio de simulação, maior produtividade agrícola se relacionou a maior rentabilidade por unidade de área. Nussio et al. (2001) citaram que a produtividade agrícola é de grande importância na competitividade de determinada opção de volumoso suplementar e cria o desafio de estruturação gerencial na propriedade no sentido de se atingir a meta de produção de forragem de acordo com aquele potencial da cultura.

\section{Conclusões}

A utilização de concentrados varia de acordo com o nível de produção e a qualidade da forrageira, portanto, a geração de renda é proporcional ao nível de produção das vacas em todas as estratégias de alimentação avaliadas. Considerando a avaliação por animal, o desempenho das estratégias de alimentação é influenciado pela qualidade das forrageiras utilizadas, enquanto, na avaliação por unidade de área, a produção de leite e a receita são fortemente influenciadas pela capacidade de suporte ou pela produtividade das forrageiras que compõem as estratégias de alimentação.

\section{Literatura Citada}

BATH, D.L.; SOSNIK, U. Formulation, delivery and inventory control of cost-effective rations. In: Van HORN, H.H.; WILCOX, C.J. (Eds.) Large dairy herd management. Savoy: American Dairy Science Association, 1992. p.709-719.

BOLETIM DO LEITE. Custo de produção da silagem de milho. ESALQ/USP-CEPEA, Piracicaba. Ano 9, nº 102, p.03, 2002. 
BOTREL, M.A.; XAVIER, D.F. Cultivares de capim-elefante para o Estado de Minas Gerais. Informe Agropecuário, v. 19, n.192, p.14-16, 1998.

CONGLETON, W.R.; EVERETT, R.W. Application of the incomplete gamma function to predict cumulative milk production. Journal of Dairy Science, v.63, p.109-119, 1980.

CÓSER, A.C; DERESZ, F.; MARTINS, C.E. Período de utilização de capim-elefante em pastagens. In: Pastejo de capim elafante. Informe Agropecuário, v.19, n.192, p.33-35, 1998.

ELY. L.O. Economics of forage programs. In: Van HORN, H.H.; WILCOX, C.J. (Eds.) Large dairy herd management. Savoy: American Dairy Science Association, 1992. p.720-730.

FERREIRA, A.H. Eficiência de sistemas de produção de leite: uma aplicação da análise envoltória de dados na tomada de decisão. Viçosa, MG: Universidade Federal de Viçosa, 2002. 120p. Dissertação (Mestrado em Economia Rural) - Universidade Federal de Viçosa, 2002.

FOX, D.G.; TYLUTKI, T.P.; TEDESCHI, L.O. et al. The net carbohydrate and protein system for evaluating herd nutrition and nutrient excretion. CNCPS version 5.0. Ithaca: Cornell University, 2003. 292p. (Model documentation).

GALAN, V.B; TORRES JR., A.M.; MATSUMOTO, R.A. Alguns aspectos dos custos de produção na pecuária de corte e leiteira. In: SIMPÓSIO SOBRE PRODUÇÃO ANIMAL, 9., 1997, Piracicaba. Anais... Piracicaba: Fundação de Estudos Agrários Luiz de Queiroz, 1997. p.161-184.

LEITE, J.L.B.; GOMES, A.T. Perspectivas futuras dos sistemas de produção de leite. In: GOMES, A.T.; LEITE, J.L.B.; CARNEIRO, A.V. (Eds.) O agronegócio do leite no Brasil. Juiz de Fora: EMBRAPA Gado de Leite, 2001. p.207-240.

MERTENS, D.R. Nonstructural and structural carbohydrates. In: Van HORN, H.H.; WILCOX, C.J. (Eds.) Large dairy herd management. Savoy: American Dairy Science Association, 1992. p.219-235.

NATIONAL RESEARCH COUNCIL - NRC. Nutrient requirements of dairy cattle. 6.ed. Washinton, D.C.: National Academic Press, 1988. 157p.

NATIONAL RESEARCH COUNCIL-NRC. Nutrient requirements of dairy cattle. 7.ed. Washinton, D.C.: National Academic Press, 2001. 381p.

NOGUEIRA, M.P. Pontos para o bom gerenciamento de uma fazenda leiteira. [S.L.]: Scott Consultoria, 2004. p.5-7. (A Nata do Leite, 76).

NUSSIO, L.G.; LIMA, L.G.; MATTOS, W.R.S. Alimentos volumosos para o período da seca. In: SIMPÓSIO SOBRE MANEJO E
NUTRIÇÃO DE GADO DE LEITE, 2001, Goiânia. Anais... Goiânia: Colégio Brasileiro de Nutrição Animal, 2001. p.85-100.

NUSSIO, L.G.; LIMA, L.G.; MATTOS, W.R.S. Planejamento da produção de alimentos para o inverno. In: SIMPOSIO SOBRE A PRODUÇÃO ANIMAL, 10., 1998, Piracicaba. Anais... Piracicaba: Fundação de Estudos Agrários Luiz de Queiroz, 1998. p.57-94.

NUSSIO, L.G.; NUSSIO, C.M.B. Aspectos técnicos e econômicos que afetam a escolha da fonte de forragem suplementar. In: SIMPÓSIO INTERNACIONAL DE PRODUÇÃO INTENSIVA DE LEITE - INTERLEITE, 6., 2003, Uberaba. Anais.. Uberaba: FMVZ-USP, 2003. p.123-135.

PEREIRA, J.C. Vacas leiteiras: aspectos práticos da alimentação. Viçosa, MG: Aprenda Fácil, 2000. 198p.

RENNÓ, F.P. Avaliação bioeconômica de estratégias de alimentação em sistemas de produção de leite. Viçosa, MG: Universidade Federal de Viçosa, 2005. 131p. Tese (Doutorado em Zootecnia) - Universidade Federal de Viçosa, 2005.

RODRIGUES, L.R.A.; RODRIGUES, T.J.D. Ecofisiologia de plantas forrageiras. In:CASTRO, P.R.C.; FERREIRA, S.O.; YAMADA, T. (Eds.) Ecofisiologia da produção agrícola. Piracicaba: POTAFÓS, 1997. p.203-227.

ROTZ, C.A.; MERTENS, D.R.; BUCKMASTER, D.R. et al. A dairy herd model for use in whole farm simulations. Journal of Dairy Science, v.82, p.2826-2840, 1999.

SCOTT CONSULTORIA. A Nata do Leite, nº58-69. Bebedouro: 2003. p.8-12.

SILVA, S.C. Condições edafo-climáticas para produção de Pannicum sp. In.: SIMPÓSIO SOBRE MANEJO DE PASTAGENS, 12. 1996, Piracicaba. Anais... Piracicaba: Fundação de Estudos Agrários Luiz de Queiroz, 1996. p.129-146.

TEDESCHI, L.O.; FOX, D.G.; PELL, A.N. et al. Development and evaluation of a tropical feed library for the Cornell Net Carbohydrate and Protein System Model. Scientia Agricola, v.59, p.01-18, 2002.

UNDERSANDER, D.J.; HOWARD, W.T.; SHEWER, R.D. Milk per acre spreadsheet for combining yield and quality into a single term. Journal of Production Agriculture, v.2, p.231-235, 1993.

VALADARES FILHO, S.C.; ROCHA JR., V.R.; CAPPELLE, E.R. Tabelas brasileiras de composição de alimentos para bovinos. Viçosa, MG: Suprema Gráfica, 2002. 297p.

Van SOEST, P.J. Nutritional ecology of the ruminant. 2.ed. Cornell University: Cornell University Press, 1994. 476p.

WALTNER, S.S.; McNAMARA, J.P.; HILLERS, J.K. Relationships of body condition score to production variables in high producing Holstein dairy cattle. Journal of Dairy Science, v.66, p.3410$3419,1993$. 